\title{
Late diagnosis of influenza in adult patients during a seasonal outbreak
}

\author{
Seong-Ho Choi ${ }^{1}$, Jin-Won Chung ${ }^{1}$, Tark Kim² ${ }^{2}$ Ki-Ho Park ${ }^{3}$, Mi Suk Lee ${ }^{3}$, and Yee Gyung Kwak ${ }^{4}$
}

\begin{abstract}
${ }^{1}$ Division of Infectious Diseases, Department of Internal Medicine, Chung-Ang University Hospital, Seoul; ${ }^{2}$ Division of Infectious Diseases, Department of Internal Medicine, Soonchunhyang University Hospital Bucheon, Bucheon; ${ }^{3}$ Division of Infectious Diseases, Department of Internal Medicine, Kyung Hee University Medical Center, Seoul; ${ }^{4}$ Division of Infectious Diseases, Department of Internal Medicine, Inje University Ilsan Paik Hospital, Goyang, Korea
\end{abstract}

Received: July 12, 2016

Revised : November 7, 2016

Accepted: November 11, 2016

\section{Correspondence to}

Jin-Won Chung, M.D.

Division of Infectious Diseases, Department of Internal Medicine, Chung-Ang University Hospital, 102 Heukseok-ro, Dongjak-gu, Seoul 06973, Korea

Tel: +82-2-6299-1420

Fax: $+82-2-825-7571$

E-mail: drjwchung@cau.ac.kr
Background/Aims: Due to advances in diagnostic techniques, clinicians are more frequently performing influenza diagnostic tests and referring to their test results ahead of the administration of neuraminidase inhibitors (NAIs). To investigate the clinical significance of the time from symptom onset to laboratory diagnosis, we reviewed the clinical characteristics of adult patients with influenza who had an early laboratory diagnosis (ED) or a late laboratory diagnosis (LD) at one of four tertiary care centers during a seasonal outbreak of influenza.

Methods: Clinical data were collected from 1,405 adults during the 2013 to 2014 influenza season. A patient was regarded as receiving an ED or LD if he/she received an influenza diagnostic test at o to 1 or 4 to 7 days after symptom onset, respectively. Early NAI therapy and late NAI therapy were defined as the administration of NAI $\leq 2$ or $>2$ days after symptom onset, respectively.

Results: Nearly half of the patients $(47.0 \%)$ received an ED ( $\mathrm{n}=661)$, whereas $13.5 \%$ $(\mathrm{n}=190)$ received a LD. Patients with a LD had initial symptoms of cough, sputum production, and dyspnea and experienced pneumonia, antibiotic therapy, hospitalization, and admission to the intensive care unit more often than those with an ED. NAI therapy and early NAI therapy were less frequent in patients with a LD than those with an ED. Of the analyzed baseline characteristics, age $\geq$ 50 years, influenza $\mathrm{B}$ infection, and diagnosis using a polymerase chain reaction test were significantly associated with a LD.

Conclusions: LD was associated with inappropriate antiviral therapy and complicated presenting features in adult patients with seasonal influenza. ED of influenza should be emphasized, especially for older adults.

Keywords: Influenza, human; Diagnosis; Antiviral agents

\section{INTRODUCTION}

Influenza virus causes community outbreaks during the winter months of both hemispheres every year. During these seasonal outbreaks, influenza virus causes acute respiratory illness in up to $20 \%$ of the population, ranging from a self-limited syndrome to serious illness resulting in hospitalization or death, especially in vulnerable patients [1]. Neuraminidase inhibitors (NAIs) have been reported to shorten symptom duration, prevent hospitalization and/or complications, and reduce mortality in patients with influenza, especially if these inhibitors are administered early in the course of the viral illness [2-4]. Thus, early administration of NAIs has been recommended for patients suspected of having influenza during a pandemic outbreak and for those who are vulnerable to influenza complications during a seasonal outbreak, even without any diagnostic testing [1]. In re- 
cent years, however, advances in diagnostic techniques have enabled clinicians to perform influenza diagnostic tests and to refer to their results ahead of the administration of NAIs more frequently than before. Few studies have examined the clinical implications of the time from symptom onset to the laboratory diagnosis of influenza in the management of influenza patients. To address this knowledge gap, we reviewed the clinical characteristics of adult patients at four tertiary care centers who had an early laboratory diagnosis (ED) or a late laboratory diagnosis (LD) of influenza. At tertiary care centers, diagnostic tests for influenza are usually performed before administration of NAIs because such tests are easily available. Moreover, precise diagnosis enables proper management of severe cases and aids in hospital infection control.

\section{METHODS}

This study was performed at four tertiary care centers in South Korea, two in Seoul (Chung-Ang University Hospital and Kyung Hee University Medical Center) and two in the province of Gyeonggi (Inje University Ilsan Paik Hospital and Soonchunhyang University Hospital Bucheon). We identified all adult patients (aged $\geq 16$ years) who visited one of the study hospitals, underwent diagnostic testing for influenza, and had a positive test result between January 1 and April 5, 2014. Infectious disease physicians at each center retrospectively reviewed patient medical records and chest radiographs. Each patient's demographics, underlying disease or condition, presenting symptoms, date of symptom onset, date of influenza diagnostic test, clinical severity, administration of NAIs, and clinical outcomes were investigated. Based on these data, patients were included in the study analyses only if (1) they had available data on the time from symptom onset to the time of influenza diagnostic testing, and (2) this time ranged from 0 to 7 days. Patients who underwent testing for influenza greater than 7 days after symptom onset were excluded because they were assumed to have inaccurately recalled the symptom onset.

Patients were defined as having an ED if they underwent an influenza diagnostic test $\leq 1$ day after symptom onset and a LD if they underwent testing $\geq 4$ days after symptom onset. Early NAI therapy and late NAI therapy were defined as the administration of NAI $\leq 2$ or $>2$ days after symptom onset, respectively. Pneumonia was defined as the presence of a new or progressive infiltrate on a chest radiograph accompanied by two or more of the following symptoms or signs: fever, cough, sputum production, dyspnea, and an attending physician's diagnosis of pneumonia.

We compared the clinical characteristics of patients with an ED to those of patients with a LD. Continuous variables were compared using Student $t$ test or the Mann-Whitney $U$ test. Categorical variables were compared using the chi-square test or Fisher exact test. A $p$ value $<0.05$ was considered significant. Logistic regression analysis was performed to identify clinical factors associated with LD. Statistical analyses were performed using SPSS version 18.0 (SPSS Inc., Chicago, IL, USA).

\section{RESULTS}

During the study period, 1,476 adult patients visited the study hospitals, underwent diagnostic testing for influenza, and had a positive test result. Patients without available data on symptom onset $(\mathrm{n}=13)$ and who underwent diagnostic testing $>7$ days after symptom onset (n =58) were excluded. Thus, 1,405 were ultimately included in the analysis.

Less than half of the study patients were male (549, $39.1 \%$ ), and the mean age was $42.9 \pm 18.5$ years. Chronic underlying disease was found in 237 patients (16.9\%), including diabetes mellitus ( $\mathrm{n}=109,7.8 \%)$, cerebrovascular disease $(n=50,3.6 \%)$, chronic lung disease $(n=49$, $3.5 \%)$, solid tumor $(n=47,3.3 \%)$, bronchial asthma $(n=43$, $3.1 \%)$, pregnancy $(n=30,2.1 \%)$, and heart failure $(n=18$, 1.3\%). Nearly half $(\mathrm{n}=661,47.0 \%)$ received an ED and 190 (13.5\%) received a LD. Laboratory diagnosis of influenza was made via the rapid influenza detection test (RIDT) in 1,338 patients (95.2\%) and via a polymerase chain reaction (PCR) test in 109 patients (7.8\%). Both tests were positive in 42 patients (3.0\%). Influenza A was detected in more than two-thirds of the study patients $(\mathrm{n}=1,017$, $72.4 \%)$, followed by influenza $B(n=384,27.3 \%)$ and co-infection with influenza A and B ( $\mathrm{n}=4,0.3 \%)$. Hospitalization, pneumonia, and admission to the intensive care unit (ICU) were observed in 295 (21.0\%), 87 (6.2\%), and 23 
(1.6\%) patients, respectively. Pneumonia was diagnosed at the initial presentation in 82 of the 87 patients with pneumonia (94.3\%). NAIs were administered to 1,246 patients $(88.7 \%)$, of whom 872 (70.0\% of 1,246$)$ received early NAI therapy. Of the 291 hospitalized patients with available outcome data, 271 survived (93.5\%), nine died in the hospital (3.1\%), and 10 were transferred to other hospitals (3.4\%).

Table 1 presents the baseline characteristics of patients with an ED and patients with a LD. The mean age of the LD group was higher than that of the ED group (48.1 years vs. 42.2 years, $p<0.001$ ). Diabetes mellitus and congestive heart failure were significantly more common in the LD group than in the ED group (12.6\% vs. $7.0 \%, p=0.01 ; 3.2 \%$ vs. $0.9 \%, p=0.03$, respectively). Chronic lung disease and liver cirrhosis also tended to be more common in the LD group ( $4.7 \%$ vs. $2.3 \%, p=$ $0.07 ; 2.1 \%$ vs. $0.6 \%, p=0.08)$. Influenza B infection was more prevalent in the LD group than in the ED group ( $41.0 \%$ vs. $21.9 \%, p<0.001$ ). Diagnosis of influenza using the PCR test was more common in the LD group $(15.8 \%$ vs. $6.7 \%, p<0.001$ ). The RIDT test result was more frequently negative in the LD group than in the ED group (21/190, $11.1 \%$ vs. $26 / 661,3.9 \%, p<0.001$ ).

Logistic regression analysis was next performed to identify clinical factors associated with LD. The model

Table 1. Baseline characteristics of adult patients with seasonal influenza who visited one of four tertiary medical centers in South Korea during spring 2014 , according to the time from symptom onset to influenza diagnostic testing

\begin{tabular}{|c|c|c|c|c|}
\hline \multirow[b]{2}{*}{ Characteristic } & \multicolumn{3}{|c|}{ Time from symptom onset to diagnostic testing } & \multirow[b]{2}{*}{$p$ value $^{\mathrm{a}}$} \\
\hline & $\begin{array}{c}\text { o-1 Day (ED group) } \\
(\mathrm{n}=661)\end{array}$ & $\begin{array}{l}2-3 \text { Days } \\
(\mathrm{n}=554)\end{array}$ & $\begin{array}{c}\text { 4-7 Days (LD group) } \\
(\mathrm{n}=190)\end{array}$ & \\
\hline Male sex & $245(37.1)$ & $233(42.1)$ & $71(37.4)$ & 0.94 \\
\hline Age, yr & $42.2 \pm 18.9$ & $42.0 \pm 17.6$ & $48.1 \pm 19.2$ & $<0.001$ \\
\hline$\geq 50$ & $217(32.8)$ & $159(28.7)$ & $88(46.3)$ & 0.001 \\
\hline$\geq 65$ & $108(16.3)$ & $78(14.1)$ & $49(25.8)$ & 0.003 \\
\hline Underlying disease or condition & $112(16.9)$ & $85(15 \cdot 3)$ & $40(21.1)$ & 0.19 \\
\hline Diabetes & $46(7 \cdot 0)$ & $39(7 \cdot 0)$ & $24(12.6)$ & 0.01 \\
\hline Cerebrovascular disease & $30(4 \cdot 5)$ & $10(1.8)$ & $10(5 \cdot 3)$ & 0.68 \\
\hline Solid tumor & $24(3 \cdot 6)$ & $19(3.4)$ & $4(2.1)$ & 0.30 \\
\hline Bronchial asthma & $23(3 \cdot 5)$ & $14(2.5)$ & $6(3.2)$ & 0.83 \\
\hline Chronic renal disease & $17(2.6)$ & $5(0.9)$ & $4(2.1)$ & 1.00 \\
\hline Chronic lung disease & $15(2.3)$ & $25(4 \cdot 5)$ & $9(4.7)$ & 0.07 \\
\hline Pregnancy & $14(2.1)$ & $10(1.8)$ & $6(3.2)$ & 0.42 \\
\hline Congestive heart failure & $6(0.9)$ & $6(1.1)$ & $6(3.2)$ & 0.03 \\
\hline Hematologic malignancy & $5(0.8)$ & $1(0.2)$ & $2(1.1)$ & 0.66 \\
\hline Dialysis & $4(0.6)$ & $3(0.5)$ & $1(0.5)$ & 1.00 \\
\hline Liver cirrhosis & $4(0.6)$ & 0 & $4(2.1)$ & 0.08 \\
\hline Type of influenza & & & & $<0.001$ \\
\hline Influenza A & $515(77 \cdot 9)$ & $391(70.6)$ & $111(58.4)$ & \\
\hline Influenza B & $144(21.8)$ & $163(29.4)$ & $77(40.5)$ & \\
\hline Co-infection of influenza A and B & $2(0.3)$ & o & $2(1.1)$ & \\
\hline Test(s) used for influenza diagnosis & & & & $<0.001$ \\
\hline Rapid influenza detection test & $617(93 \cdot 3)$ & $519(93.7)$ & $160(84.2)$ & \\
\hline PCR test & $26(3.9)$ & $20(3.6)$ & $21(11.1)$ & \\
\hline Both tests & $18(2.7)$ & $15(2.7)$ & $9(4.7)$ & \\
\hline
\end{tabular}

Values are presented as number (\%) or mean \pm SD.

ED, early laboratory diagnosis; LD, late laboratory diagnosis; PCR, polymerase chain reaction.

${ }^{\mathrm{a}} \mathrm{ED}$ vs. LD. 
Table 2. Clinical features, management, and outcomes of adult patients with seasonal influenza who visited one of four tertiary medical centers in South Korea during spring 2014, according to the time from symptom onset to influenza diagnostic testing

\begin{tabular}{|c|c|c|c|c|}
\hline \multirow[b]{2}{*}{ Characteristic } & \multicolumn{3}{|c|}{ Time from symptom onset to diagnostic testing } & \multirow[b]{2}{*}{$p$ value $^{a}$} \\
\hline & $\begin{array}{c}\text { o-1 Day (ED group) } \\
(\mathrm{n}=661)\end{array}$ & $\begin{array}{c}2-3 \text { Days } \\
(\mathrm{n}=554)\end{array}$ & $\begin{array}{c}\text { 4-7 Days (LD group) } \\
(\mathrm{n}=190)\end{array}$ & \\
\hline \multicolumn{5}{|l|}{ Presenting symptom } \\
\hline Cough & $528 / 660(80.0)$ & $484(87 \cdot 4)$ & $166(87.4)$ & 0.02 \\
\hline Sputum production & $337 / 660(51.1)$ & $339(61.2)$ & $121(63.7)$ & 0.002 \\
\hline Rhinorrhea & $312 / 660(47 \cdot 3)$ & $248(44.8)$ & $92(48.4)$ & 0.78 \\
\hline Sore throat & $347 / 660(52.6)$ & $293(52.9)$ & $96(50.5)$ & 0.62 \\
\hline Dyspnea & $37 / 660(5.6)$ & $39(7 \cdot 0)$ & $28(14.7)$ & $<0.001$ \\
\hline Fever & $601 / 660(91.1)$ & $499(90.1)$ & $155(81.6)$ & $<0.001$ \\
\hline Myalgia & $419 / 660(63.5)$ & $325(58.7)$ & $98(51.6)$ & 0.003 \\
\hline \multicolumn{5}{|l|}{ Clinical severity } \\
\hline Hospitalization & $126(19.1)$ & $114(20.6)$ & $55(28.9)$ & 0.003 \\
\hline Admission to intensive care unit & $6(0.9)$ & $10(1.8)$ & $7(3 \cdot 7)$ & 0.01 \\
\hline Pneumonia & $28(4.2)$ & $28(5.1)$ & $31(16.3)$ & $<0.001$ \\
\hline \multicolumn{5}{|l|}{ Treatment } \\
\hline Oxygen supplementation & $29 / 660(4.4)$ & $25(4 \cdot 5)$ & $15(7 \cdot 9)$ & 0.06 \\
\hline Use of neuraminidase inhibitor & $595(90.0)$ & $495(89.4)$ & $156(82.1)$ & 0.003 \\
\hline Within 2-day after the onset of symptoms & $589 / 595(99.0)$ & $279 / 495(56.4)$ & $4 / 156(2.6)$ & $<0.001$ \\
\hline Within 2-day after diagnostic testing & $590 / 595(99.2)$ & $493 / 495(99.6)$ & $152 / 156(97 \cdot 4)$ & 0.10 \\
\hline Antibiotic therapy & $119(18.0)$ & $104(18.8)$ & $48(25 \cdot 3)$ & 0.03 \\
\hline Outcomes of hospitalized patients & & & & 0.95 \\
\hline Survived & $118 / 126(93.7)$ & $106 / 113(93.8)$ & $48 / 5^{2}(92.3)$ & \\
\hline Died in hospital & $4 / 126(3.2)$ & $3 / 113(2.7)$ & $2 / 52(3.8)$ & \\
\hline Transferred to another hospital & $4 / 126(3.2)$ & $4 / 113(3 \cdot 5)$ & $2 / 5^{2}(3.8)$ & \\
\hline
\end{tabular}

Values are presented as number (\%).

ED, early laboratory diagnosis; LD, late laboratory diagnosis.

${ }^{\mathrm{a}} \mathrm{ED}$ vs. LD.

included sex, age, diabetes, chronic heart failure, type of influenza, and type of diagnostic test. Age $\geq 50$ years (adjusted odds ratio [OR], 1.49; 95\% confidence interval [CI], 1.05 to $2.11 ; p=0.03$ ), influenza B infection (adjusted OR, 2.01; 95\% CI, 1.52 to $2.90 ; p<0.001$ ), and diagnosis using the PCR test (adjusted OR, 2.18; 95\% CI, 1.34 to 3.56; $p=$ 0.002) were independently associated with LD.

Table 2 presents the clinical features and outcomes of the ED and LD groups. In terms of presenting symptoms, cough $(87.4 \%$ vs. $80.0 \%, p=0.02)$, sputum production $(63.7 \%$ vs. $51.1 \%, p=0.002)$, and dyspnea ( $14.7 \%$ vs. $5.6 \%, p<0.001)$ were more commonly observed in the LD group than in the ED group. However, both fever and myalgia were more common in the ED group (91.1\% vs. $81.6 \%, p<0.001 ; 63.5 \%$ vs. $51.6 \%, p=0.003)$. Hospitalization (28.9\% vs. $19.1 \%, p=0.003)$, pneumonia $(16.3 \%$ vs. $4.2 \%, p<0.001$ ), ICU admission ( $3.7 \%$ vs. $0.9 \%, p=$ 0.01 ), and receipt of antibiotic therapy (25.3\% vs. 18.0\%, $p$ $=0.03$ ) were more commonly observed in the LD group than in the ED group. However, NAI therapy (82.1\% vs. $90.0 \%, p=0.003)$ and early NAI therapy (2.6\% vs. $99.0 \%$, $p<0.001$ ) were less frequently received in the LD group than in the ED group. The outcomes of hospitalized patients were similar in terms of in-hospital mortality rate (LD vs. ED; $3.8 \%$ vs. 3.2\%). 


\section{DISCUSSION}

During a seasonal influenza outbreak, $47.0 \%$ and $13.5 \%$ of the study patients had an ED or a LD, respectively. LDs were more commonly observed in patients who were older than 50, had influenza B infection, and were diagnosed via the PCR test. Adult patients with influenza with a LD presented with clinical features of pneumonia and received antibiotic therapy more frequently than patients with an ED. However, NAI therapy and early NAI therapy were both performed less frequently in patients with a LD.

The clinical significance of LD has not been reported. Our study identified a few characteristic features of adult patients with a LD of influenza. First, patients with a LD had lower probabilities of NAI therapy and early NAI therapy than those with an ED (Table 2). Thus, LD might be associated with inappropriate patient management, especially patients particularly vulnerable to the complications of influenza. From a public health perspective, during the uninterrupted period of viral shedding caused by the absence of early NAI therapy, patients with a LD can transmit their viruses to a large number of uninfected people. Second, patients with a LD had pneumonia at initial presentation and received antibiotic therapy more frequently than patients with an ED. Moreover, LD was associated with more frequent hospitalization and ICU admission. This finding can be explained by the smaller likelihood that patients with uncomplicated influenza will visit the hospital and therefore are less likely to undergo diagnostic testing after 4 days have passed from symptom onset compared to patients with complicated influenza. Thus, complicated presenting features might be more commonly found in patients with a LD than in those with a ED. Additionally, healthcare costs can be increased in patients with a LD, mainly due to their more frequent antibiotic therapy and more intensive in-hospital care. Considering these clinical features associated with LD, ED of influenza should be emphasized during each influenza season.

Despite their relatively mild clinical features, nearly half of the study patients $(47.0 \%$ of the 1,405$)$ received a laboratory diagnosis and management o to 1 days after symptom onset at the four tertiary care centers. In South Korea, many tertiary care centers are concentrated in Seoul and Gyeonggi province and are easily accessible to patients with relatively mild medical problems. Additionally, the centers compete on economic grounds to attract patients. Patients with uncomplicated influenza can be safely managed at primary or secondary clinics during a seasonal outbreak. Only a small percentage of these patients need to visit tertiary care centers for further evaluation and management. Thus, the optimal role of primary and secondary clinics in the management of seasonal influenza in South Korea might warrant further evaluation.

A LD can result from at least one of the following causes: (1) late presentation of a patient to medical care; (2) late performance of an influenza diagnostic test; (3) a false negative test result at the initial stage; and (4) delayed reporting of the test result. In this study, old age was independently associated with LD. Elderly patients are more likely to have less prominent initial signs and symptoms of infectious disease compared to younger adults [5]. Thus, this finding can be explained by the first two causes of LD discussed above, i.e., late presentation and late testing. On the other hand, early presentation and early diagnostic testing could be more frequent in younger adults than in older ones, because younger adults might be more vigilant of the development of influenza than older ones due to their active social roles in the workplace and/or concerns of transmitting influenza to others. The finding that the RIDT is less sensitive in detecting influenza B than influenza A can explain why influenza B was associated with a LD [6]. Since a diagnosis using the RIDT was more common in the ED group than in the LD group, fewer cases of influenza B might have been diagnosed in the ED group, resulting in more influenza B cases in the LD group. The more frequent diagnosis using PCR in the LD group could be a result of the low sensitivity of the RIDT in the late stage of influenza infection, when viral shedding is reduced compared to the early stage $[1,7]$. Thus, patients whose presentation was delayed after symptom onset should undergo the RIDT and PCR tests simultaneously rather than the RIDT alone.

This study had several important limitations. First, because early NAI therapy is sometimes recommended without any laboratory diagnostic test, the clinical implication of the time from symptom onset to laboratory diagnosis might not be generalizable to all clinical situations. Second, information regarding patients' preced- 
ing visits to primary or secondary clinics was not available. Unknown clinical interventions might have been performed for some of the study patients before their presentation to the study hospitals, although it is likely that these occurred in only a small number of the study patients. Third, due to the retrospective nature of the study, data were not collected on influenza vaccination.

In conclusion, LD of influenza was associated with inappropriate antiviral therapy and complicated presenting features in adult patients with seasonal influenza. Since appropriate management of influenza now relies heavily on early laboratory diagnosis, early diagnosis of influenza should be emphasized, especially for older adults.

\section{KEY MESSAGE}

1. These $13.5 \%$ of adult patients received a late laboratory diagnosis of influenza at four tertiary care centers during 2013 to 2014 influenza season.

2. Late diagnosis of influenza was associated with inappropriate antiviral therapy and complicated presenting features in adults with seasonal influenza.

3. Early diagnosis of influenza should be emphasized especially for older adults.

\section{Conflict of interest}

No potential conflict of interest relevant to this article was reported.

\section{REFERENCES}

1. Harper SA, Bradley JS, Englund JA, et al. Seasonal influenza in adults and children: diagnosis, treatment, chemoprophylaxis, and institutional outbreak management: clinical practice guidelines of the Infectious Diseases Society of America. Clin Infect Dis 2009;48:1003-1032.

2. Dobson J, Whitley RJ, Pocock S, Monto AS. Oseltamivir treatment for influenza in adults: a meta-analysis of randomised controlled trials. Lancet 2015;385:1729-1737.

3. Muthuri SG, Venkatesan S, Myles PR, et al. Effectiveness of neuraminidase inhibitors in reducing mortality in patients admitted to hospital with influenza A HıNıpdmog virus infection: a meta-analysis of individual participant data. Lancet Respir Med 2014;2:395-404.

4. Muthuri SG, Myles PR, Venkatesan S, Leonardi-Bee J, Nguyen-Van-Tam JS. Impact of neuraminidase inhibitor treatment on outcomes of public health importance during the 2009-2010 influenza $\mathrm{A}\left(\mathrm{H}_{1} \mathrm{~N}_{1}\right)$ pandemic: a systematic review and meta-analysis in hospitalized patients. J Infect Dis 2013;207:553-563.

5. Crossley KB, Peterson PK. Infections in the elderly. In: Bennett JE, Dolin R, Blaser MJ, eds. Mandell, Douglas, and Bennett's Principles and Practice of Infectious Diseases. 8th ed. Philadelphia (PA): Elsevier Saunders, 2014:3459-3465.

6. Chartrand C, Leeflang MM, Minion J, Brewer T, Pai M. Accuracy of rapid influenza diagnostic tests: a meta-analysis. Ann Intern Med 2012;156:500-511.

7. Chan MC, Lee N, Ngai KL, Leung TF, Chan PK. Clinical and virologic factors associated with reduced sensitivity of rapid influenza diagnostic tests in hospitalized elderly patients and young children. J Clin Microbiol 2014;52:497501. 